\title{
Delay driven spatiotemporal chaos in single species population dynamics models
}

\author{
Masha Jankovic, Sergei Petrovskii\# \\ Department of Mathematics, University of Leicester, \\ Leicester, U.K. \\ and Malay Banerjee \\ Department of Mathematics and Statistics, IIT Kanpur, India
}

\begin{abstract}
Questions surrounding the prevalence of complex population dynamics form one of the central themes in ecology. Limit cycles and spatiotemporal chaos are examples that have been widely recognised theoretically, although their importance and applicability to natural populations remains debatable. The ecological processes underlying such dynamics are thought to be numerous, though there seems to be consent as to delayed density dependence being one of the main driving forces. Indeed, time delay is a common feature of many ecological systems and can significantly influence population dynamics. In general, time delays may arise from interand intra-specific trophic interactions or population structure, however in the context of single species populations they are linked to more intrinstic biological phenomena such as gestation or resource regeneration. In this paper, we consider theoretically the spatiotemporal dynamics of a single species population using two different mathematical formulations. Firstly, we revisit the diffusive logistic equation in which the per capita growth is a function of some specified delayed argument. We then modify the model by incorporating a spatial convolution which results in a biologically more viable integro-differential model. Using the combination of analytical and numerical techniques, we investigate the effect of time delay on pattern formation. In particular, we show that for sufficiently large values of time delay the system's dynamics are indicative to spatiotemporal chaos. The chaotic dynamics arising in the wake of a travelling population front can be preceded by either a plateau corresponding to dynamical stabilisation of the unstable equilibrium or by periodic oscillations.
\end{abstract}

\# Corresponding author. E-mail: sp237@le.ac.uk 


\section{Introduction}

Factors and mechanisms determining the spatial population distribution of ecological species are a major focus of interest in ecology (Fortin and Dale 2005, Ritchie 2010). Often the distribution shows remarkable spatial variability, which is usually referred to as patchiness (Levin 1994, Rietkerk et al. 2004), where areas or 'patches' of high population density are separated from areas where the given species is either present at a very low density or is absent altogether. A classical example of such a patchy spatial distribution is given by plankton (Levin and Segel 1976, Martin 2003), although terrestrial species, in particular insects, often exhibit considerable spatial variability as well (Liebhold et al. 2013).

One obvious explanation of this phenomenon is swarming behaviour (Okubo 1986), especially when it is reinforced by social interactions between the animals (e.g. Mogilner and EdelsteinKeshet 1999, Mogilner et al. 2003) and/or by taxis (Tyutyunov et al. 2004, 2009). On a larger spatial scale, an intuitive explanation of the spatial heterogeneity in species distribution lies in environmental variability, e.g. spatial distribution is being driven by the heterogeneous distribution of resources (Liebhold et al. 1994, Grünbaum 2012). However, in many cases this does not seem to be the case as the observed population distributions appear to be largely uncorrelated with the environment (Powell et al. 1975, Sharov et al. 1997). The heterogeneous spatial population distribution can also arise as a result of biotic interactions. In particular, there is a large body of literature showing, both theoretically and empirically, that a spatial pattern can be a result of predator-prey or host-parasite interactions (Hassell et al. 1991, Hastings et al. 1997, Davis et al. 1998, Petrovskii and Malchow 1999, Jankovic and Petrovskii 2013), or multi-specific competition (Petrovskii et al. 2001, Adamson and Morozov 2012, Mimura and Tohma 2014). Note that, in the case of biotic-driven pattern formation, the patterns can be regarded as selforganised, i.e. they are not related to any external forcing; in fact, they can arise in a uniform environment.

Self-organised pattern formation is often related to instability of the spatially uniform distribution; a well-known example is given by the Turing instability (Segel and Jackson 1972, Klausmeier 1999). A necessary condition of the Turing instability is the differing diffusivity of interacting species; in order to make the instability practically observable (i.e. to occur in a reasonably broad range of population dynamics' parameters), the diffusion coefficients have to be at least an order of magnitude apart. This large difference in the mobility of a prey and its predator does not often happen, and hence the ecological importance of the Turing scenario of pattern formation is rather limited (but see Sherratt 2013).

An alternative mechanism is sometimes referred to as biological turbulence ${ }^{1}$ or a "wave of chaos" (Petrovskii and Malchow 2001) which becomes possible when the dynamics of the interacting species are oscillatory. From a theoretical perspective, this is usually related to the existence of a stable limit cycle, e.g. see Turchin (2003). The properties of the population's spatial distribution emerging due to this mechanism were shown to be in agreement with field observations (Medvinsky et al. 2002, Petrovskii et al. 2003, Malchow et al. 2008). However, the capacity of biological turbulence to explain patchiness of ecological populations is somewhat limited too, as the existence of the limit-cycle population oscillations requires interaction of the

\footnotetext{
${ }^{1}$ The term "biological turbulence" was suggested to Sergei Petrovskii by Lutz Schimansky-Geier in a private discussion in 1999.
} 
given species (prey) with its specialist predator (cf. Rosenzweig 1971, May 1972). Specialist predators are relatively rare in nature and that may explain why genuine predator-prey cycles are not often seen ${ }^{2}$. As long as the predator is a generalist, in a realistic multi-species community predator-prey cycles are unlikely to occur, as the changes in the density of prey become uncoupled from that of the predator because of the complicated switching behaviour of the latter (Comins and Hassell 1976, Holt 1983, Morozov and Petrovskii 2013, Van Leeuwen et al. 2013). Heterogeneous population distribution is therefore a far more general phenomenon than the theoretical mechanisms that have by far been brought forward as its explanation.

There is, however, another feature of population dynamics as ubiquitous as species heterogeneity, and this is time delay. Delayed density dependence is thought to be one of the main factors causing population fluctuations (Berryman and Turchin 1997). The most commonly considered causal mechanisms are resource competition (Hansen et al. 1998), cannibalism (Briggs et al. 2000), and maternal effects (Ginzburg and Taneyhill 1994) where the nutritional environment of the parental generation can influence the growth and reproductive potential of the next generation. Delays may also occur as a consequence of developmental time and/or interaction between individuals of different stages (Royama 1981, Hastings 1984). In mathematical terms, the destabilisation of a positive steady state, both in nonspatial and spatial systems, usually occurs through the Hopf bifurcation (Green and Stech 1981, Fowler 1982, Busenberg and Huang 1996, Li et al. 2008, Su et al. 2009) that leads to limit-cycle oscillatory behaviour. We menton here that such destabilization does not always happen; in particular, if the population growth is damped by a strong Allee effect, an increase in time delay does not necessarily lead to the Hopf bifurcation, e.g. see Jankovic and Petrovskii (2014).

In this paper, we examine the inherent relation between these two phenomena, i.e. time delays and pattern formation. We are especially interested in the possibility of the onset of spatiotemporal chaos and, respectively, the formation of irregular spatial patterns. We mention here that, whilst the dynamics of time-delayed nonspatial systems are understood relatively well, time-delayed spatial systems pose a much bigger challenge. Although there is a large body of literature concerned with time-delayed spatially explicit population dynamics (e.g. see the references above), the vast majority of it is concerned with either a travelling front or a periodic pattern (Ashwin et al. 2002, Yoshida 1982, Su et al. 2009). Meanwhile, in population systems where limit cycles appear for other reasons (i.e. not related to time delay), travelling waves and periodic patterns are known to be only a part of the rich spectrum of spatiotemporal dynamics (Petrovskii and Malchow 2000) that, in particular, may exhibit chaotic oscillations (Sherratt et al. 1995, Sherratt 2001, Petrovskii et al. 2001). Correspondingly, the possibility of spatiotemporal pattern formation and chaos in a single species population with time delay is our main interest here. We first consider pattern formation triggered by travelling population fronts in a heuristic delayed diffusion-reaction equation where the delay is included into the per capita growth, and reveal the onset of spatiotemporal chaos in the wake of the front. We then consider a somewhat more realistic model where the delay is appropriately spatially averaged (Britton 1990, Gourley and Bartuccelli 1995, Gourley and Chaplain 2002, Ashwin et al. 2002), which results in an integro-differential model, and show that it exhibits qualitatively similar properties such as, in

\footnotetext{
${ }^{2}$ The famous hare-lynx cycle (Elton and Nicholson 1942, May 1975) is sometimes regarded as the only available example, although there are different opinions on that (cf. Sherratt and Smith 2008) and the discussion is by no means over.
} 
particular, the onset of chaos.

The remainder of the paper is organised as follows. In the following section (Section 2) we introduce our modelling framework and revisit known analytical results for the stability condition and loss of monotonicity conditions for both Hutchinson's equation and the diffusive logistic equation. We then investigate through numerical simulations the effect time delay has on the diffusive logistic model (Section 3). In Section 4, we introduce a modified model and discuss the concept and reasoning behind the spatial averaging, as well as presenting numerical results. Section 5 summarises our findings.

\section{Modelling framework and some analytical results}

Temporal dynamics of a single species popuation can be described by the following generic ordinary differential equation:

$$
\frac{d U}{d t}=U f(U)
$$

where $U$ is the population size (density) and $f(U)$ describes the per capita growth. For many populations the observed biological reality imposes a saturation level, known as the carrying capacity, which forms a numerical upper bound on growth. Correspondingly, the simplest form of such, the Verhulst-Pearl logistic equation is frequently used to model self-limiting populations:

$$
\frac{d U}{d t}=r U\left(1-\frac{U}{K}\right)
$$

where $r$ is the intrinsic rate of growth and $K$ is the carrying capacity. Logistic growth implies rapid initial growth at low population densities due to local aggregation and a nearly exponential decay to the population's carrying capacity due to the negative feedback through intraspecific competition. Admittedly a simple model, such behaviour is in qualitative agreement with observed dynamics of many populations, especially under laboratory, resource-limited, conditions. In nature, though, events do not often occur instantaneously as predicted by the above model. For this reason, Hutchinson (1948) suggested a more appropriate and biologically sound model including a time delay accounting for resource regeneration, postulating that the available resources affecting specific growth rates of populations, depend on the population density $(U)$ at some point $\tau$ in the past where $\tau$ is the development time of the resource. From Hutchinson's (1948) seminal paper and thereafter, time delay models have gained significance and become a much studied topic in mathematical ecology. The model proposed is a delay differential equation:

$$
\frac{d U}{d t}=r U\left(1-\frac{U_{\tau}}{K}\right)
$$

where $\tau>0$ is the time delay, and $U_{\tau}=U(t-\tau)$. Even though the choice of delayed mechanism still remains questionable, the above model was used extensively to model natural populations with reasonable success, see May (1975).

Aiming to incorporate both spatial and temporal population dynamics, the model is therefore given by a delay reaction-diffusion equation:

$$
\frac{\partial U}{\partial t}=D \frac{\partial^{2} U}{\partial x^{2}}+r U\left(1-\frac{U_{\tau}}{K}\right)
$$


where $D$ is the diffusion coefficient. In what follows, we present both analytical and numerical results to investigate the effect of time delay in Eq. (2.4). We consider Eq. (2.4) in the context of biological invasion (Shigesada and Kawasaki 1997), so that the 'initial' population distribution $U(x, t)$ for $-\tau<t<0$ is assumed to be a function of compact support.

It is often thought that the addition of diffusive terms will not change much the system's dynamical structure, cf. Huang (1998). However, below we will show that this is not necessarily true and that a spatial model can exhibit dynamical regimes that are not possible in its nonspatial counterpart. Whilst the nonspatial Hutchinson's model is only capable of exhibiting periodic oscillations, its spatial counterpart can display chaos. Some of our results will then be extended, using numerical simulations, to include the corresponding 2D case:

$$
\frac{\partial U}{\partial t}=D\left(\frac{\partial^{2} U}{\partial x^{2}}+\frac{\partial^{2} U}{\partial y^{2}}\right)+r U\left(1-\frac{U_{\tau}}{K}\right)
$$

where we also demonstrate the onset of chaotic oscillations.

Lastly, we consider an alternative formulation which includes a spatial convolution to account for the continual movement of individuals and the time it takes them to move. In particular, the 'correct' average population density is now assumed to be a spatial (and in general, temporal) average weighted towards the current position of individuals. This follows from the observation that competition does not occur instantaneously. The resulting model is an integrodifferential equation, for which we show that despite previous studies on similar models, the onset of spatiotemporal chaos is possible.

\subsection{Linear stability analysis}

It is worth noting that single ordinary differential equations, unlike delay differential equations (DDEs), cannot exhibit oscillatory, limit cycle behaviour (see Fig. 2.1). While qualitative features of DDEs for population growth dynamics near bifurcation points, including analytical solutions, may be found (Fowler 1982), the easiest (and often the only) way to investigate the quantitative properties is by numerical simulations, that are straightforward to make. In this section, we first revisit some results of the linear stability analysis to obtain the stability condition on $\tau$. We then further our study by obtaining the loss of monotonicity condition for the travelling front solution of Eq. (2.4), cf. Ashwin et al. (2002).

We begin by recalling the spatially homogeneous Hutchinson's equation which will serve as the baseline model. Stability is examined only for the positive steady state $U_{*}=K$, as the extinction steady state, $U_{*}=0$, is always unstable. We nondimensionalise the equation, thereby decreasing the number of parameters (Murray 2002):

$$
\frac{d \widetilde{U}(\tilde{t})}{d \widetilde{t}}=\widetilde{U}(\widetilde{t})(1-\widetilde{U}(\widetilde{t}-\widetilde{\tau}))
$$

where $\widetilde{U}=\frac{U}{K}, \widetilde{t}=r t$ and $\widetilde{\tau}=r \tau$. Suppose $\widetilde{u}$ is a small perturbation from the steady state $\widetilde{U_{*}}=1$, such that:

$$
\widetilde{u}=\widetilde{U}-\widetilde{U_{*}} .
$$

By linearising, and omitting higher order terms, we obtain the following equation:

$$
\frac{d \widetilde{u}(\tilde{t})}{d \widetilde{t}} \approx-\widetilde{u}(\widetilde{t}-\widetilde{\tau}),
$$



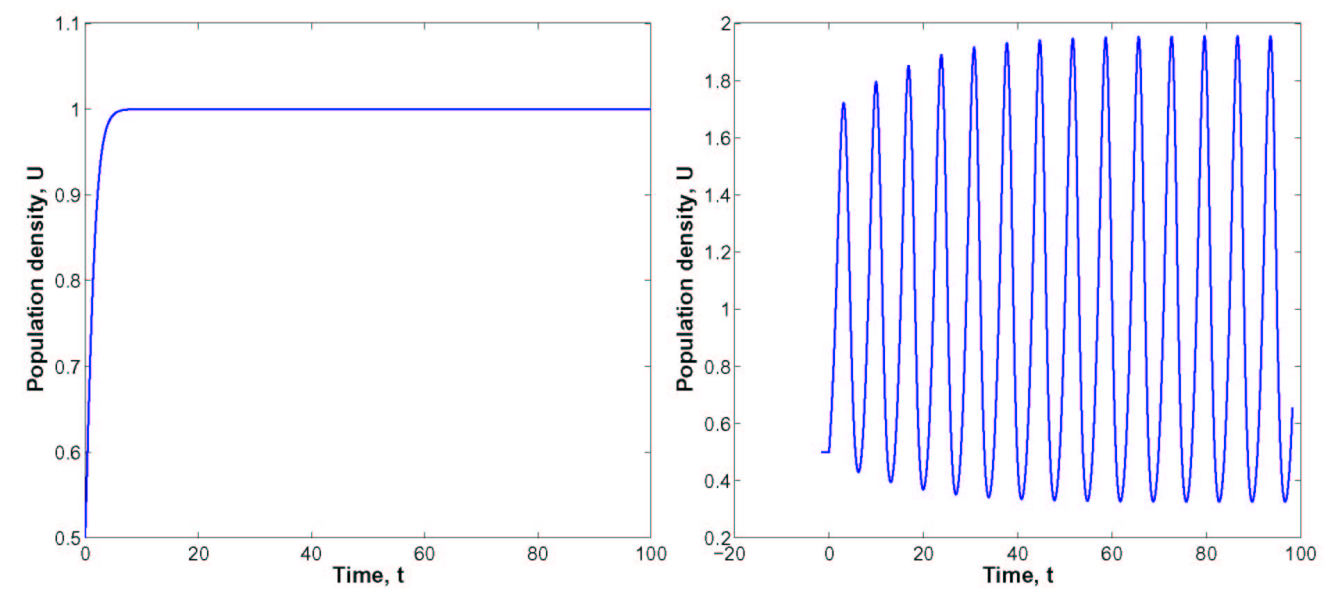

Figure 2.1: Snapshots of solution behaviour for the instantaneous logistic equation (left) and Hutchinson's equation (right), with delay parameter $\tau=1.7$. Other parameters are $r=1$ and $K=1$.

for which solutions must be of exponential form $\widetilde{u}(\widetilde{t})=c e^{\lambda \widetilde{t}}$, where $c$ is a constant and $\lambda$ are the eigenvalues that, when substituted into Eq. (2.8), lead to the transcendental characteristic equation:

$$
\lambda=-e^{-\lambda \widetilde{\tau}}
$$

so that $\lambda$ is a function of the (dimensionless) delay $\tilde{\tau}$.

The equilibrium point $\widetilde{U_{*}}=1$ is asymptotically stable if all eigenvalues have negative real parts. For this reason we set $\lambda=\mu+\imath \omega$, substitute into Eq. (2.8), and separate the real and imaginary parts:

$$
\begin{aligned}
& \mu=-e^{-\mu \widetilde{\tau}} \cos \omega \widetilde{\tau}, \\
& \omega=e^{-\mu \widetilde{\tau}} \sin \omega \widetilde{\tau} .
\end{aligned}
$$

Considering two cases, when the eigenvalues are real and complex, we determine the stability condition on $\widetilde{\tau}$. It is readily seen that, in case of real eigenvalues (i.e. $\omega=0) \mu$ cannot take positive value, thus the steady state is always stable. If eigenvalues are complex, the stability condition reads $\mu<0$ therefore implying $\omega \widetilde{\tau}<\pi / 2$. The Hopf bifurcation point is found for $\mu(\widetilde{\tau})=0$, thus $\omega \widetilde{\tau}_{H}=\pi / 2$. From Eq. (2.10) the only viable solution is $\omega=1$, hence $\widetilde{\tau}_{H}=\pi / 2$. Scaling back to dimensional quantities, the equilibrium $U_{*}=K$ is stable if $0<r \tau<\pi / 2$, and unstable otherwise $(r \tau>\pi / 2)$.

Stability conditions obtained for Hutchinson's equation can be easily extended to the diffusive logistic equation and verified by simulations, which will be done in the following sections. Fig. 2.2 shows the bifurcation diagrams for both cases. Note that, whilst the nonspatial case described by Eq. (2.6) exhibits only limit cycle behaviour for overcritical values of $\tau$, the dynamics of the spatiotemporal system (Eq. (2.4), or (3.20) in dimensionless variables) is obviously more complicated indicating chaotic dynamics. We consider this issue in detail in Section 3. 

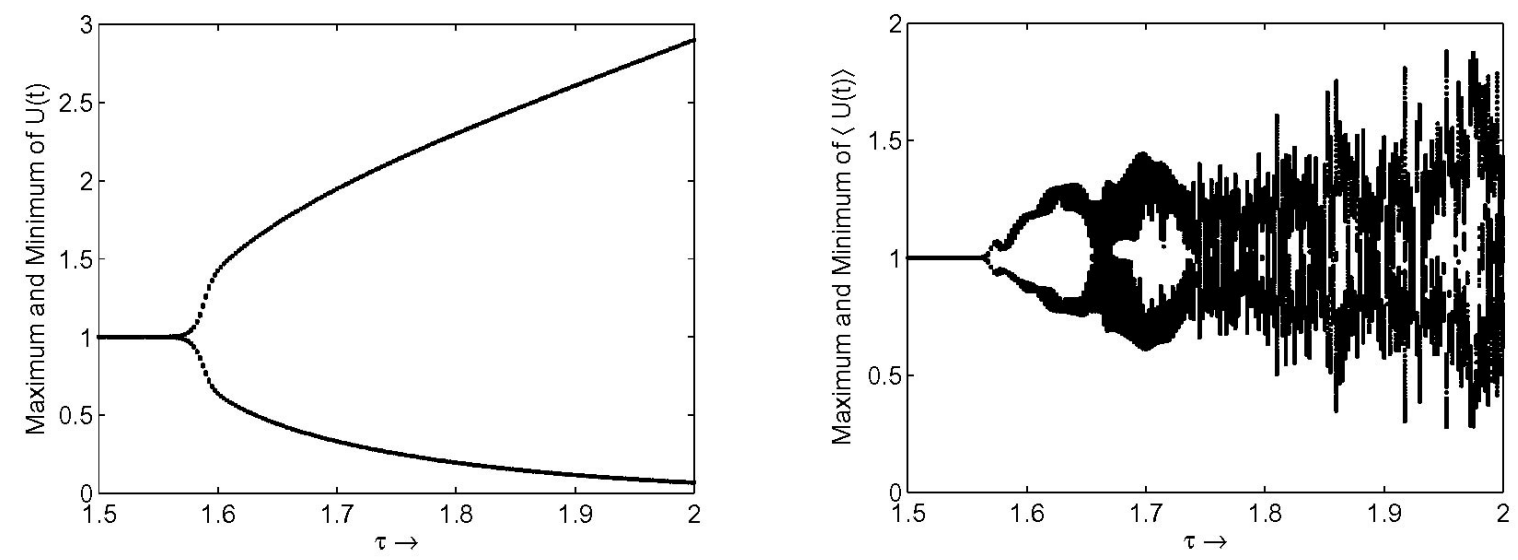

Figure 2.2: Bifurcation diagrams for Hutchinson's equation (left) and for the delay diffusion equation (right) with $\tau$ being the bifurcation parameter.

\subsection{Loss of monotonicity}

Before the positive steady state loses its stability, another change in the solution's properties takes place, namely, the loss of monotonicity. Let us rewrite Eq. (2.9) for the eigenvalues as a function of both $\lambda$ and $\tilde{\tau}$ :

$$
F(\lambda ; \tilde{\tau})=\lambda+e^{-\lambda \tilde{\tau}}=0 .
$$

Obviously, function $F$ has a unique minimum and it is readily seen that, when $\tilde{\tau}$ is sufficiently small, this minimum lies in the third quarter of the $(\lambda, F)$ plane, so that Eq. (2.12) has two real negative roots. The positive steady state is stable and the solution approaches it monotonously, e.g. as can be seen from the left panel of Fig. 2.1. When $\tilde{\tau}$ increases, the minimum is pushed upwards; correspondingly, the two roots move towards each other so that finally, for some $\tilde{\tau}=\tilde{\tau}_{*}$, they merge and 'disappear', which actually means that they become complex. For $\tilde{\tau}=\tilde{\tau}_{h}>\tilde{\tau}_{*}$, the Hopf bifurcation takes place, but for the values of $\tilde{\tau}$ just slightly larger than $\tilde{\tau}_{*}$ the real part of the eigenvalues is still negative. It means that the positive steady state is still stable but the solution approaches it in an oscillatory manner.

More quantitatively, in order to obtain the value of $\tilde{\tau}_{*}$, one needs to consider Eq. (2.12) together with the tangency condition:

$$
\frac{\partial F}{\partial \lambda}=1-\tau e^{-\lambda \widetilde{\tau}}=0 .
$$

Having solved the system (2.12-2.13), one obtains that $\tilde{\tau}_{*}=1 / e$ (e.g. Yoshida 1982). In original dimensional parameters, it means that solutions of Hutchinson's equation start oscillating for $r \tau>1 / e$.

We now consider how the loss of monotonicity condition can be extended and applied to Eq. (2.4). A compact initial population distribution is known to converge to two travelling fronts propagating in opposite directions with the same speed $c$, i.e. $U(x, t) \rightarrow \widetilde{U}(x-c t) \cup \widetilde{U}(x+c t)$. Consider, for instance, the front propagating to the right, $U(x, t)=\widetilde{U}(\xi)$ where $\xi=x-c t$, which 
is the solution of the following equation (in dimensionless variables):

$$
\frac{d^{2} \widetilde{U}(\xi)}{d \xi^{2}}+c \frac{d \widetilde{U}(\xi)}{d \xi}+\widetilde{U}(\xi)(1-\widetilde{U}(\xi+c \tau))=0
$$

corresponding to the conditions at infinity as $\widetilde{U}(\xi) \rightarrow 0$ for $\xi \rightarrow \infty$ and $\widetilde{U}(\xi) \rightarrow 1$ for $\xi \rightarrow-\infty$.

If we linearise around the steady state $\widetilde{U}_{*}=1$, by setting $\widetilde{U}=1+\widetilde{v}$, where $\widetilde{v}$ is a small perturbation, it gives a second order differential equation:

$$
\widetilde{v}_{\xi \xi}+c \widetilde{v}_{\xi}-\widetilde{v}(\xi+c \tau)=0 .
$$

As before, we try a solution of exponential form $\widetilde{v}=e^{\lambda \xi}$ and obtain the characteristic equation:

$$
F(\lambda ; \tau)=\lambda^{2}+c \lambda-e^{\lambda c \tau} .
$$

When the delay is zero, the eigenvalue equation has two real roots of opposite sign. By increasing the time delay the positive root branches into two roots, which get closer to each other and eventually coalesce, then turn complex. Loss of monotonicity is associated with the loss of real positive eigenvalues and this coalescence which occurs at a critical value, $\tau_{c r}$; see Fig. 2.3. We denote the double root itself, $\lambda_{*}$, and it must satisfy both:

$$
F\left(\lambda_{*} ; \tau_{c r}\right)=0 ; \quad \frac{\partial F}{\partial \lambda}\left(\lambda_{*} ; \tau_{c r}\right)=0 .
$$

Following some standard calculations, one can find the loss of monotonicity condition as the following implicit expression (Ashwin et al. 2002):

$$
\frac{2}{c \tau}+\sqrt{\frac{4}{c^{2} \tau^{2}}+c^{2}}=c \tau \exp \left(1+\sqrt{1+\frac{c^{4} \tau^{2}}{4}}-\frac{c^{2} \tau}{2}\right) .
$$

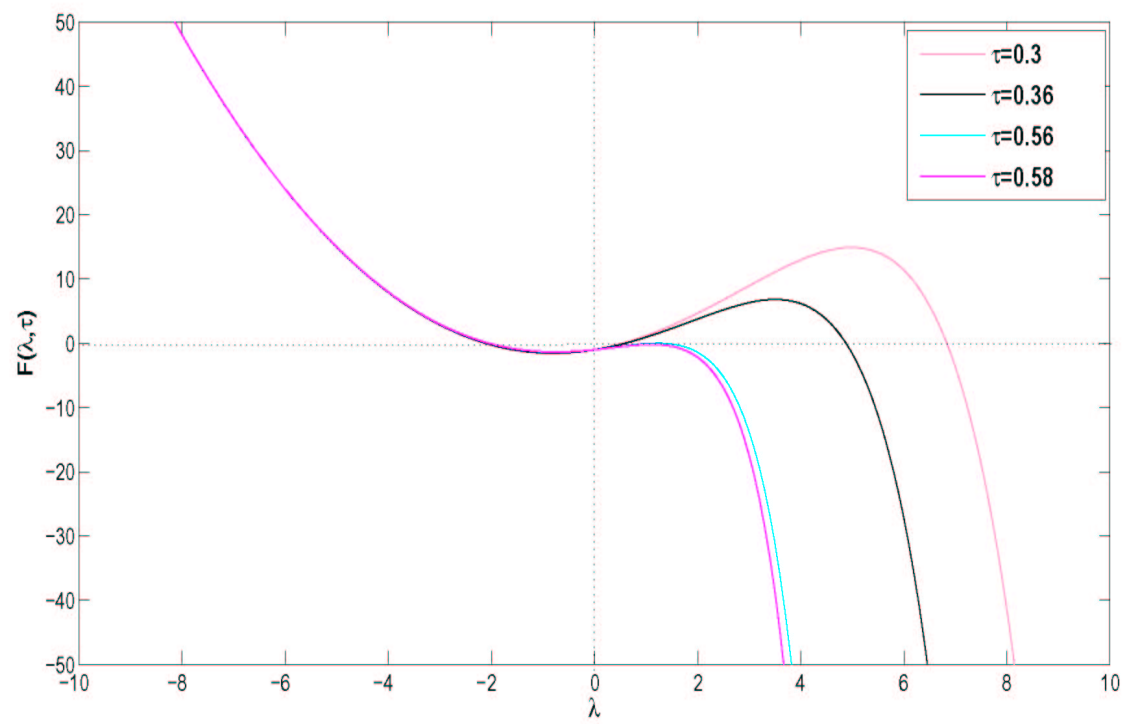

Figure 2.3: Plot of $F(\lambda ; \tau)$ for different values of $\tau$. The critical value, when loss of monotonicity occurs, is calculated as $\tau_{c r} \approx 0.5608$ for the minimum wave speed $c=2$. 
For any given value of the front speed $c$, Eq. (2.18) determines the critical value of $\tau$. We now notice that the travelling wave equation (2.14) linearised in the vicinity of the extinction state $\widetilde{U}_{*}=0$, i.e. at the leading edge of the front, coincides with the linearised Fisher equation, and hence the same argument can be used resulting in the condition $c \geq 2$. Indeed, compact initial conditions are known to converge to a travelling front propagating with the minimum speed $c=2$ (e.g. Ashwin et al. 2002). In this case, Eq. (2.18) takes a somewhat simpler form:

$$
\frac{1}{\tau}+\sqrt{\frac{1}{\tau^{2}}+4}=2 \tau e^{1+\sqrt{1+4 \tau^{2}}-2 \tau}
$$

Equation $(2.19)$ is solved numerically resulting in $\tau_{c r} \approx 0.5608$.

\section{Simulations}

Apart from the results discussed above, it is difficult to establish other solution properties analytically, so we have to resort to simulations. We begin with the 1D diffusive logistic equation, Eq. (2.4). We write it in dimensionless variables:

$$
\frac{\partial U}{\partial t}=\frac{\partial^{2} U}{\partial x^{2}}+U\left(1-U_{\tau}\right)
$$

and solve it numerically by finite-differences for different values of the time delay $\tau$, hence considering $\tau$ as the controlling parameter. The mesh steps, $\Delta t$ and $\Delta x$, were taken to be sufficiently small, ensuring accuracy and reliability of our results. The spatial domain is chosen to be sufficiently large in order to minimise the impact of boundaries. At the domain boundaries, the Neumann type 'no flux' conditions are used.

The initial condition is used in the following form, which is partially inspired by the problem of biological invasion (cf. Sherratt et al. 1995, Shigesada and Kawasaki 1997):

$$
U(x, 0)=U_{0} \quad \text { if } \quad|x| \leq \frac{L}{2}, \quad \text { otherwise } \quad U(x, 0)=0,
$$

where $U_{0}>0$ is the initial population density and $L$ determines the size of the initially occupied patch. Since Eq. (3.20) involves a time delay, we supplement the initial condition with the population's 'history', i.e. the data on $U(x, t)$ for $-\tau<t<0$. For this purpose we choose a constant function equal to the initial population density:

$$
U(x, t)=U_{0} \quad \forall \quad t \in(-\tau, 0) .
$$

Typical simulation results obtained in the model (3.20) for different values of the time delay are shown in Figs. 3.4-3.7. Since the mathematical problem is symmetric with respect to the origin, only the right-hand half of the domain is shown. We therefore observe that, for a sufficiently small time delay $\left(\tau_{c r}<\tau<\pi / 2\right.$ ), the initial conditions evolve into a propagating population front with damped oscillations at the front $^{3}$, see Fig. 3.4. Behind the front, the population density promptly converges to the spatially uniform steady state $U(x, t)=1$. The damped oscillations

\footnotetext{
${ }^{3}$ Note that for smaller values of time delay, $0<\tau<\tau_{c r}$, i.e. before the monotonicity condition (2.19) is broken, the front is monotone. We do not show this case here for the sake of brevity.
} 
at the front tend to grow in amplitude with increasing $\tau$ and can become prominent but decay promptly behind the front.

However, a further increase in $\tau$ alters the solution's behaviour significantly. As the system surpasses the Hopf bifurcation, apparent spatially irregular oscillations emerge in the wake of
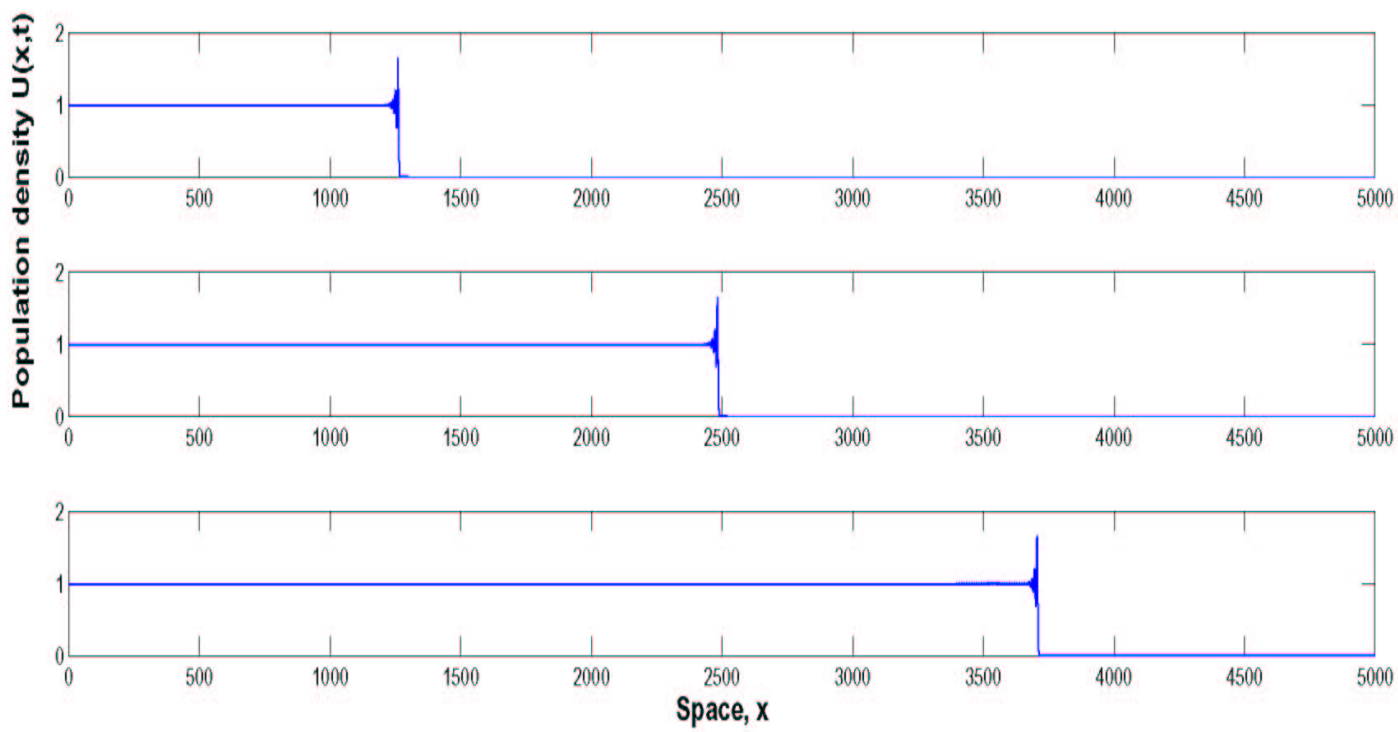

Figure 3.4: Snapshots of the population density (simulated with Eq. (3.20)) shown at equidistant moments $t=1, t=667, t=1333$ and $t=1999$ for the time delay $\tau=1.5$. Other parameters are $U_{0}=0.5$ and $L=100$.
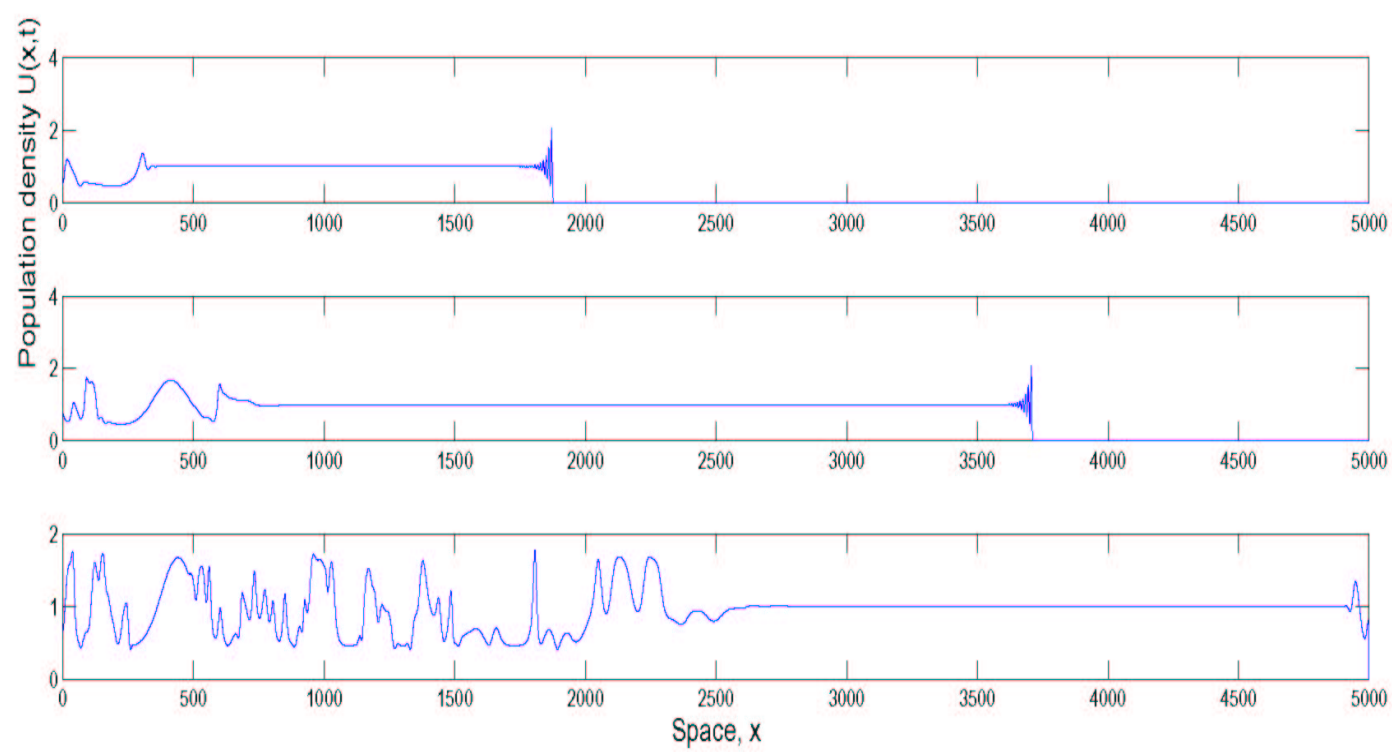

Figure 3.5: Snapshots of the population density at equidistant moments $t=1, t=1001, t=2000$ and $t=2999$ as obtained in Eq. (3.20) for $\tau=1.7$. Other parameters are the same as in Fig. 3.4. 
the propagating front; see Figs. 3.5 and 3.6. Connecting the two dynamically differing scenarios, chaos in the wake and damped oscillations at the leading edge, a quasi-homogeneous plateau appears. This plateau is nontrivial as it corresponds to the system's unstable equilibrium point $U_{*}=K$. Qualitatively similar dynamics of the dynamical stabilisation of unstable equilibrium were observed in systems of coupled instantaneous reaction-diffusion equations, e.g. predator-prey models (Sherratt et al. 1995, Petrovskii and Malchow 2000, Malchow and Petrovskii 2002) and a three-species competition model (Petrovskii et al. 2001). The length of the plateau increases with simulation time, see Fig. 3.5. The dynamical stabilisation is a spatiotemporal feature that cannot be directly linked to dynamics in the corresponding nonspatial system. As far as we are aware, this type of dynamics is usually attributed to multi-species models, and is a novel concept in the context of single species population models. We emphasise here that the patterns emerging in the wake of the plateau (e.g. see the bottom panel in Fig. 3.5 and the two middle panels in Fig. 3.6) are purely self-organised; they are not induced by any spatial heterogeneity in the system as there is no heterogeneity (the parameters are space-independent). The irregular spatiotemporal patterns are self-sustained; following the onset of apparent chaos, no further qualitative changes in the system's dynamics occur at a later time. For longer simulation time, as the fronts propagate, the aperiodic oscillations eventually occupy the whole numerical domain; e.g. see the bottom of Fig. 3.6.

By even further increasing the controlling parameter, $\tau$, one would expect more prominent oscillatory behaviour, as can indeed be observed (cf. Figs. 3.6 and 3.7). Interestingly, for a sufficiently large $\tau$, instead of the quasi-homogeneous distribution, a wave-train of periodic oscillations is formed behind the propagating population front; see Fig. 3.7. Our simulations indicate that these periodic oscillations appear abruptly without any gradual transition. A slight difference in $\tau$ results in a different pattern, cf. Figs. 3.6 and 3.7. This seems to suggest that the

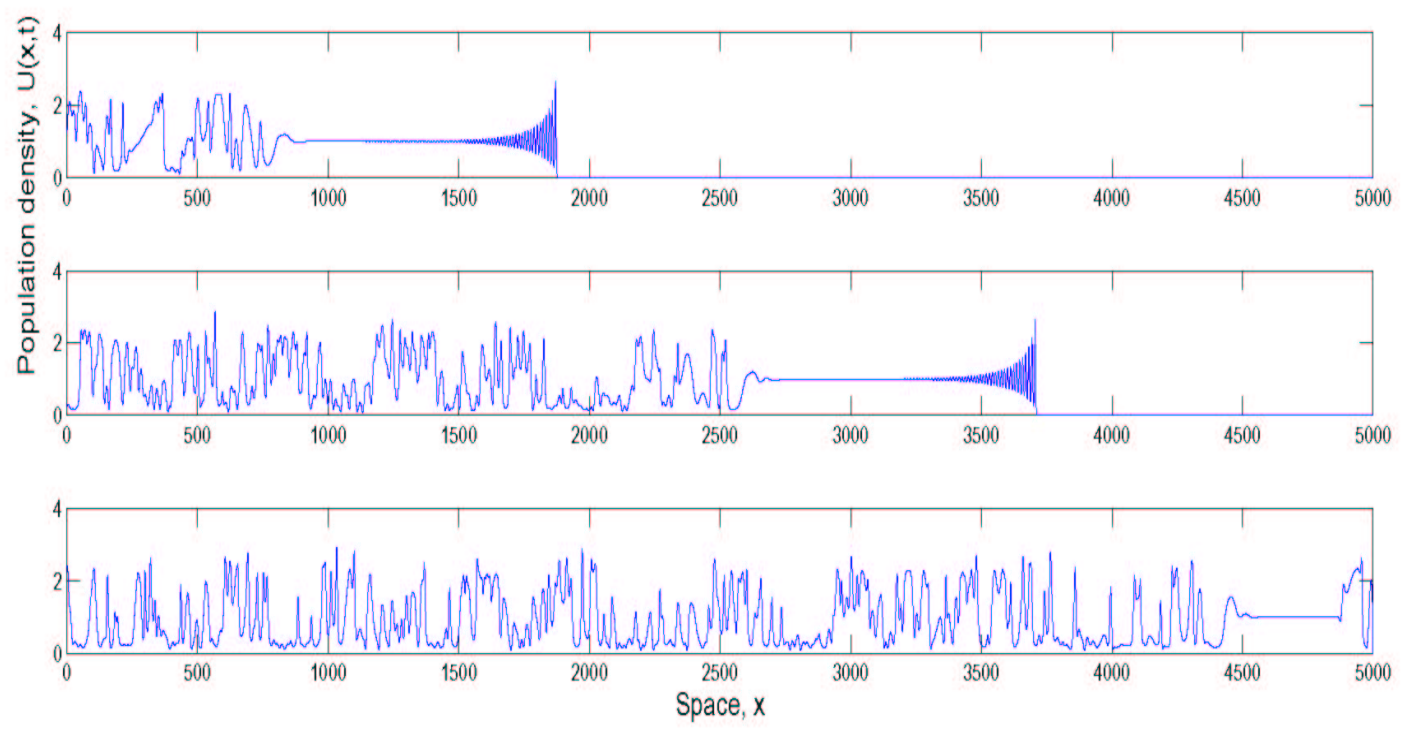

Figure 3.6: Snapshots of the population density at equidistant moments $t=2, t=1001, t=2000$ and $t=2999$ obtained for $\tau=1.94$. Other parameters are the same as in Fig. 3.4. 

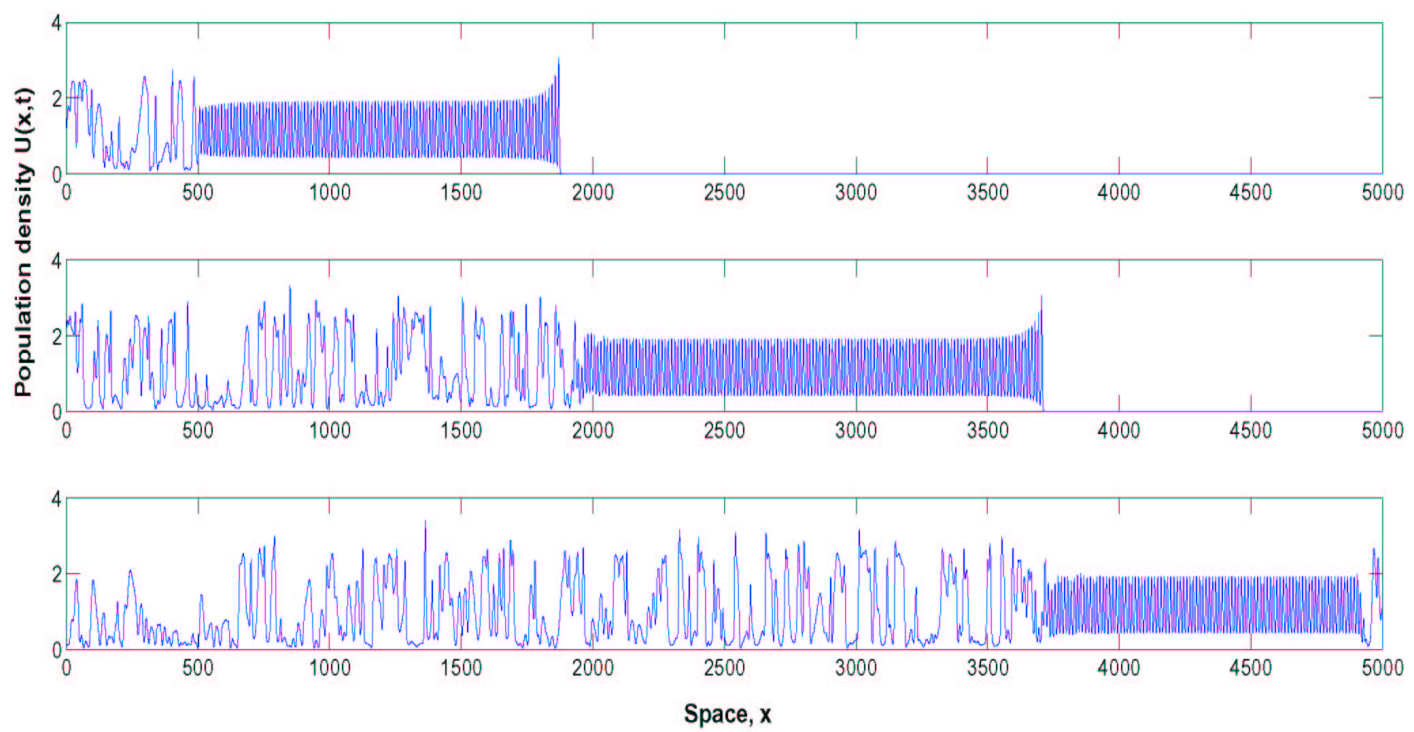

Figure 3.7: Snapshots of the population density at equidistant moments $t=2, t=1001, t=2000$ and $t=2999$ obtained for $\tau=1.95$. Other parameters are the same as in Fig. 3.4.

sudden change in dynamics should not be attributed to the Hopf bifurcation. We mention that a further increase in $\tau$ (not shown here for the sake of brevity) does not lead to any qualitative changes: in fact, even the amplitude of the periodic oscillations shows almost no change.

We have also investigated the corresponding temporal dynamics of the system, see Fig. 3.8. The population density was recorded at the mid-point of the spatial numerical domain and clearly exhibits irregular oscillations. This result is in contrast to the temporally periodic oscillations observed by Ashwin et al. (2002) for the equivalent system.

In order to verify that the irregular spatiotemporal patterns are chaotic, we tested the sensitivity of solutions to initial conditions. Figure 3.9 shows spatial propagation of two population fronts with slightly different initial conditions. As simulation time is increased, small perturbations grow and induce large differences between the two solutions. Since such sensitivity is known to be a characteristic property of chaos, this leads to the conclusion that the dynamics shown in Figs. 3.5-3.9 is indeed chaotic.

Chaotic behaviour is also apparent in the phase plane $\left(U\left(x_{*}, t\right), U\left(x_{*}, t-\kappa \tau\right)\right)$, see Fig. 3.10, where the axes show population densities at the same location $x_{*}$ but at different time moments, being separated by a certain time lag which is defined as a fraction $\kappa$ of the time delay $\tau$. The trajectories are intricate and indicate complex behaviour of solutions. Note that, by decreasing the time lag $\kappa$, the trajectory in the phase plane tends to shrink to the bisector line. Correspondingly, the oscillations in the population density become highly correlated; however, behaviour still remains chaotic.

We have also investigated the corresponding spatiotemporal pattern formation scenario in the 2D case. Figure 3.11 shows snapshots of the population density obtained in the model (2.5) (in dimensionless variables $D=r=K=1$ ) for $\tau=1.9>\tau_{H}$, i.e. in the parameter range where the $1 \mathrm{D}$ system exhibits the onset of spatiotemporal chaos. Since the system is symmetric 


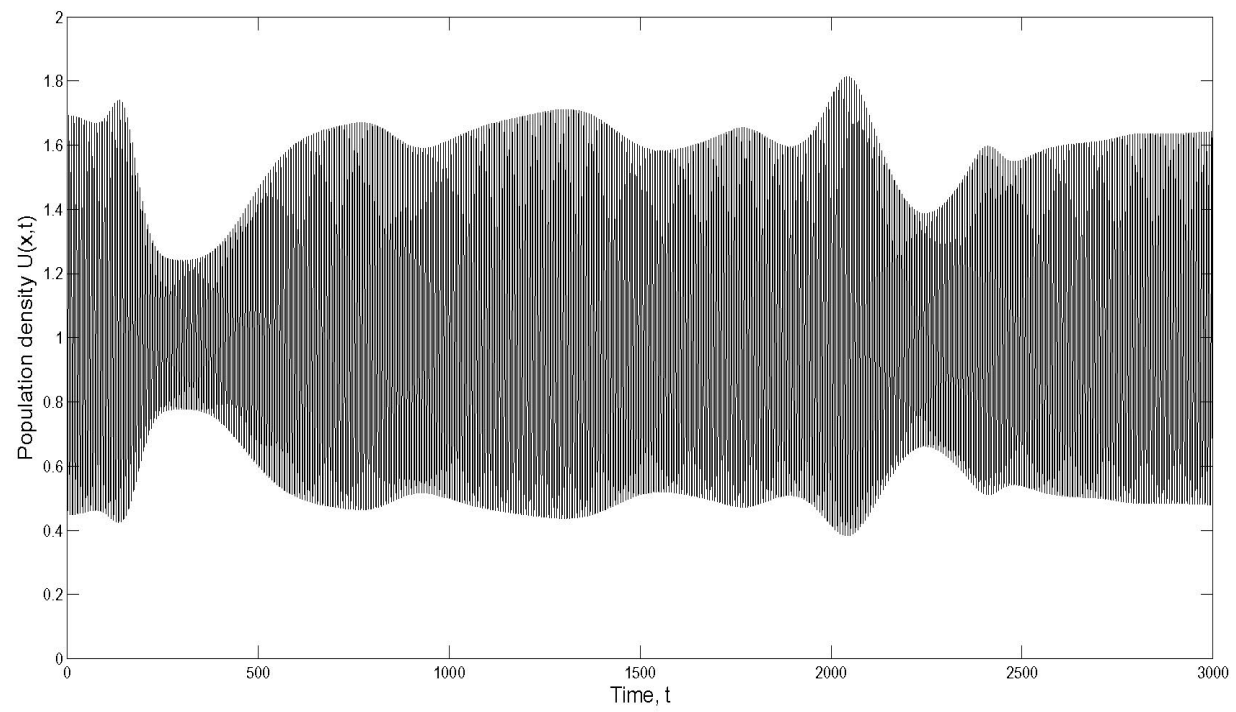

Figure 3.8: Population density vs time at a fixed location, i.e. $U\left(x_{*}, t\right)$ where $x_{*}=2500$, shown after the irregular oscillations occupied the whole domain. Here the black shape is made of many high-frequency oscillations that visually almost merge at this resolution. The irregular variations in the amplitude of the oscillations indicate chaotic dynamics. The delay is set to $\tau=1.7$, other parameters are the same as above.

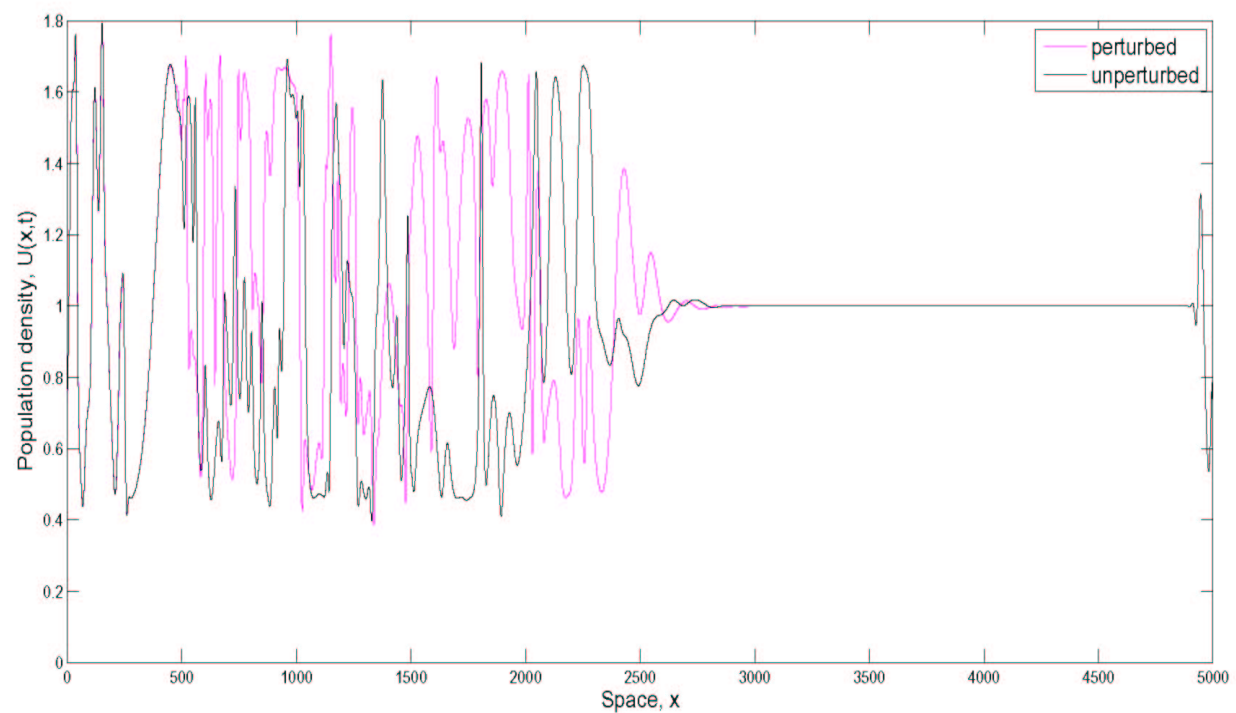

Figure 3.9: Snapshots of two solutions to Eq. (3.20) with $\tau=1.7$ obtained from slightly different initial conditions. In one case $U_{0}=0.5$ ('unperturbed solution'), in the other case $U_{0}=0.502$ ('perturbed solution').

with regards to reflection $(x \rightarrow-x$ and $y \rightarrow-y)$, simulation results are shown only in the first 

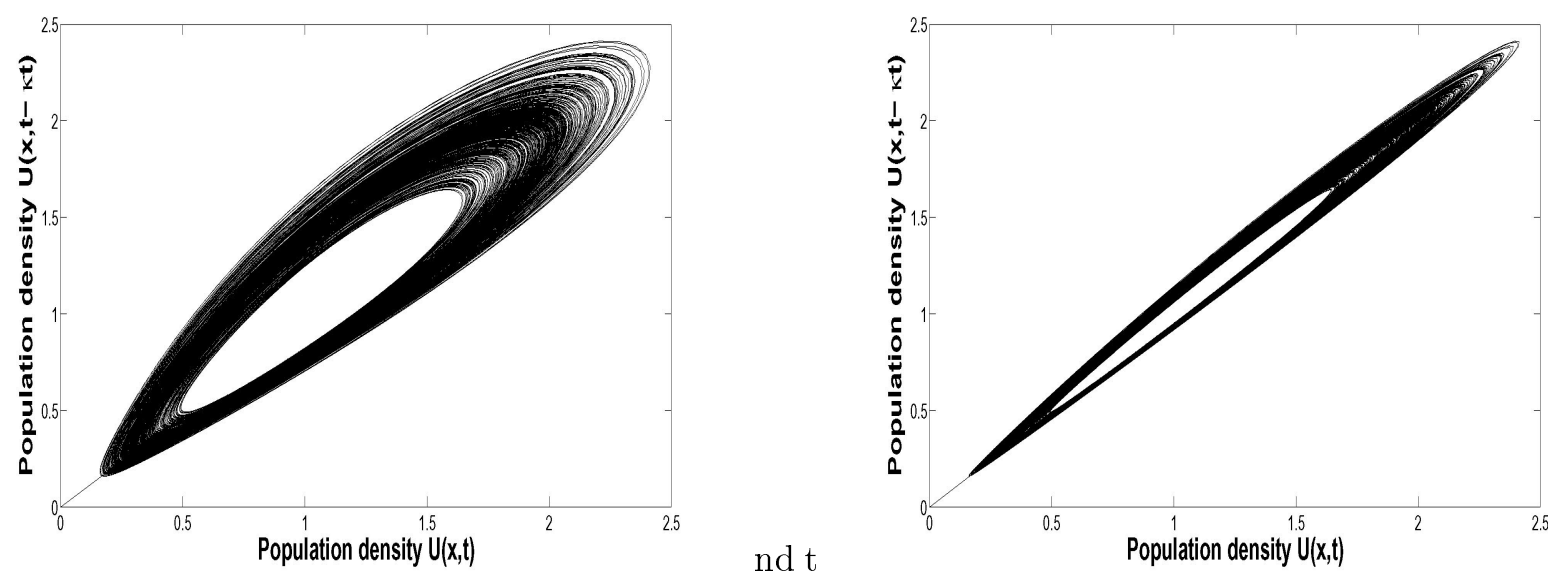

Figure 3.10: Phase plane of Eq. (3.20) where axes show population densities at the same location in space but at different moments, i.e. separated by a time lag defined as a fraction $\kappa$ of the time delay, the latter being chosen as $\tau=1.7$. By decreasing the time lag between solutions a higher correlation is observed; left for $\kappa=1 / 4$, right for $\kappa=1 / 16$.

quadrant. It is readily seen that the $2 \mathrm{D}$ dynamics mirrors the dynamics of the corresponding $1 \mathrm{D}$ model. Namely, the travelling population front exhibits fast decaying oscillations at the front. Damped oscillations are followed by a plateau emerging due to the dynamical stabilisation of the unstable equilibrium. For a larger simulation time, irregular spatiotemporal patterns emerge in the wake of the front. These chaotic oscillations develop gradually and eventually spread throughout the numerical domain. Patchiness in the wake is therefore an inherent property of the delayed system and is induced by a sufficiently large time delay. As before, the patterns are triggered by the population fronts themselves, not by any prescribed heterogeneity, i.e. the patchiness is self-organised and self-sustained.

\section{Modified model: spatial averaging}

As we have mentioned in the introduction, the spatial version of Hutchinson's model (Eq. 2.4) has been a focus of several studies previously, e.g. see Busenberg and Huang (1996), Fowler (1982), Huang (1998), although spatiotemporal chaos was not reported. However, it has been increasingly recognised (cf. Britton 1990, Gourley and Chaplain 2002) that the straightforward inclusion of diffusion into delay equations such as Eq. (3.20) is perhaps not biologically relevant. The underlying assumption of diffusion is that individuals freely and randomly move around, without any preferred direction. Importantly, this movement of individuals takes time which needs to be accounted for. For this reason, the 'true' average population density should be a spatiotemporal average weighted towards both current time and positioning of individuals (Britton 1990). The idea, proposed by Britton (1990), is to calculate the probability of an individual having been at position $y$ at some previous time $t-\tau$, given that it is at point $x$ at current time $t$, by summing across all previous times and positions:

$$
\iint P(\text { at } y \text { at time } t-\tau \mid \text { at } x \text { at } t) U(y, t-\tau) \omega(\tau) \delta y \delta \tau,
$$



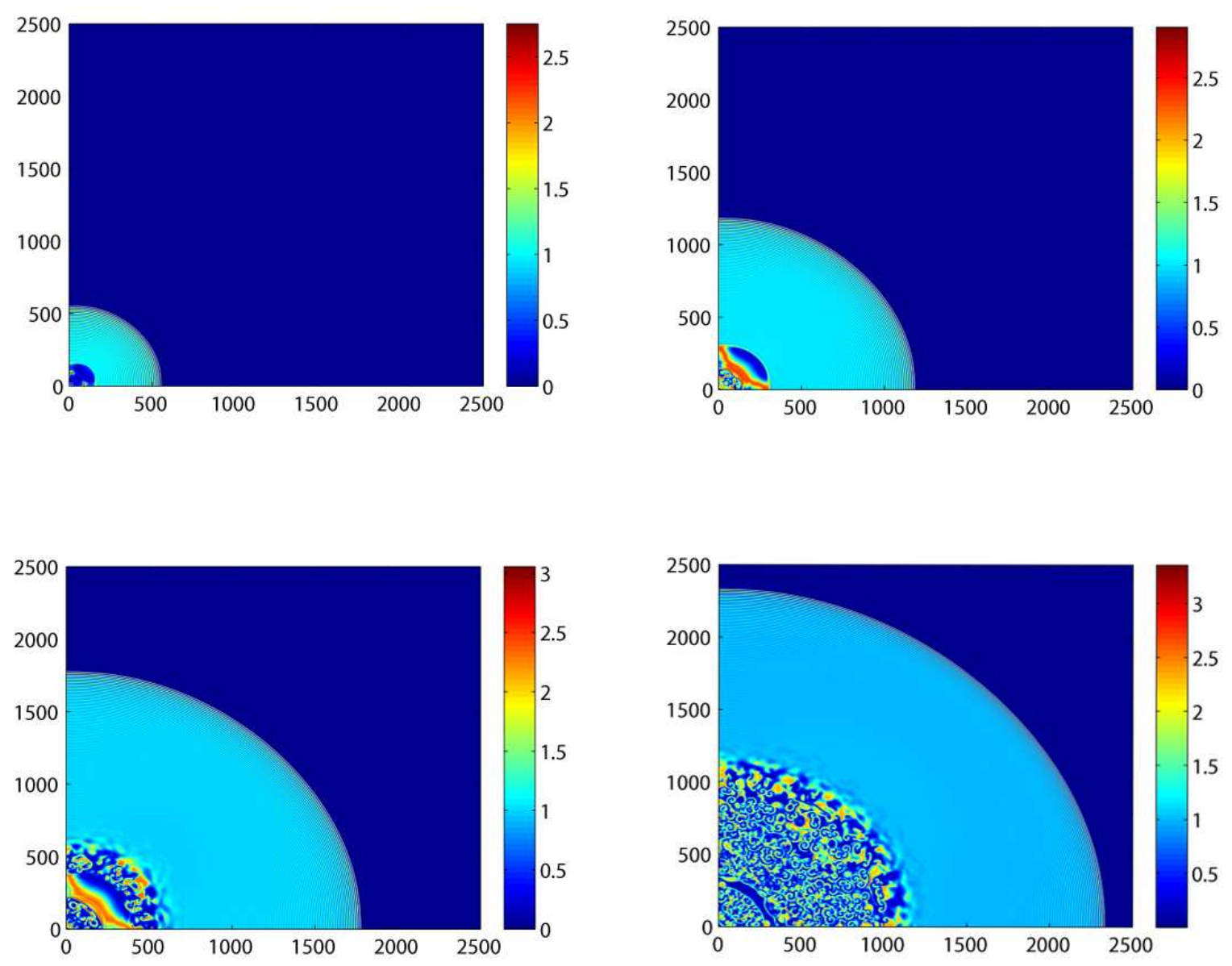

Figure 3.11: Snapshots of spatial propagation of population front in two dimensions as described by Eq. (2.5). Snapshots are given for simulation times: $t=280, t=600, t=940$ and $t=1280$ (left to right, top to bottom). The time delay is $\tau=1.9$ and other parameters are $\Delta t=0.1$, $\Delta x=\Delta y=1, D=1, K=1, r=1, U_{0}=0.5$.

where $P$ is a probability density function, $U$ is the population density and $\omega$ is a weighting function determining the importance of previous times $(t-\tau)$ on the current time $t$. Obviously, the choice of a particular probability function is linked to the nature of the movement. In case of diffusion, the probability density is given (in continuous space and time) by the following function:

$$
P(\text { at } y \text { at time } t-\tau \mid \text { at } x \text { at } t)=\frac{1}{\sqrt{4 \pi \tau}} e^{\frac{-|x-y|^{2}}{4 \tau}},
$$

in dimensionless variables (effectively, assuming the diffusion coefficient being equal to unity). As we are interested in a discrete time delay, the weighting function $\omega(\tau)$ will be the $\delta$ function, $\omega(\tau)=\delta(t-\tau)$. Therefore, in our case we consider only the spatial average through integrating over all possible previous positions; hence, our improved model reads as follows:

$$
\frac{\partial U}{\partial t}=D \frac{\partial^{2} U}{\partial x^{2}}+r U\left(1-\frac{1}{K} \int_{-\infty}^{\infty} \frac{1}{\sqrt{4 \pi \tau}} e^{\frac{-(x-y)^{2}}{4 \tau}} U(y, t-\tau) d y\right)
$$




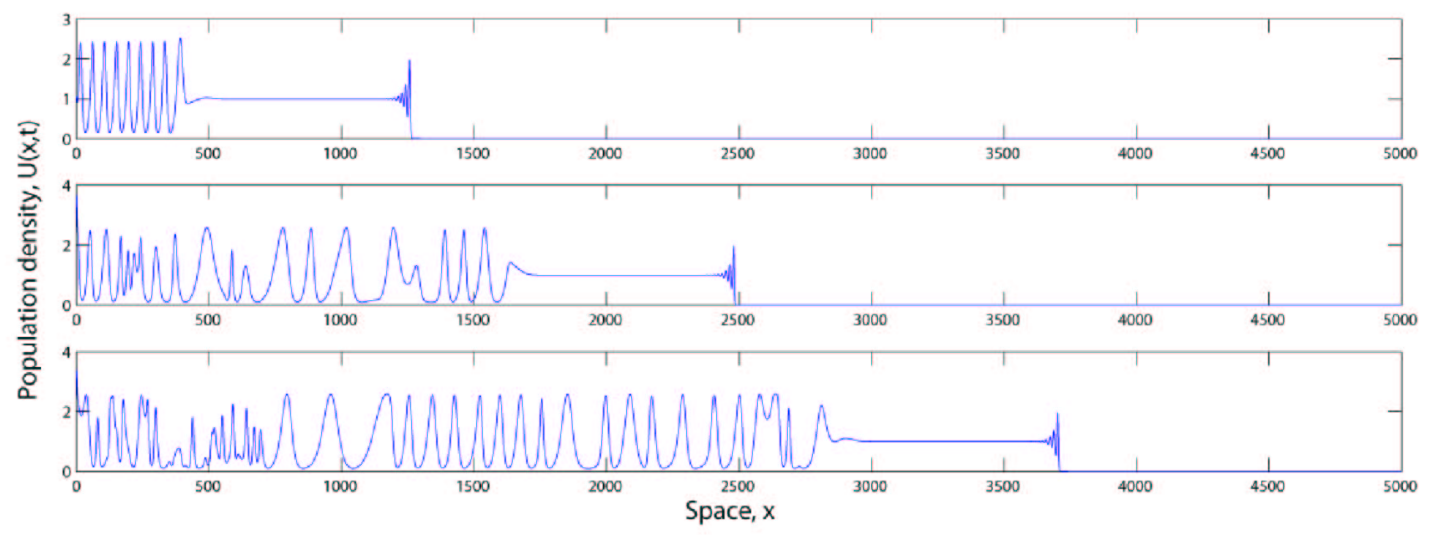

Figure 4.12: Snapshots of spatial propagation of population front with spatial averaging taken at equidistant moments in time: $t=2665, t=3331$ and $t=3997$. The time delay parameter is chosen to be 1.95 , and other parameters are $r=1, K=1, L=100$.

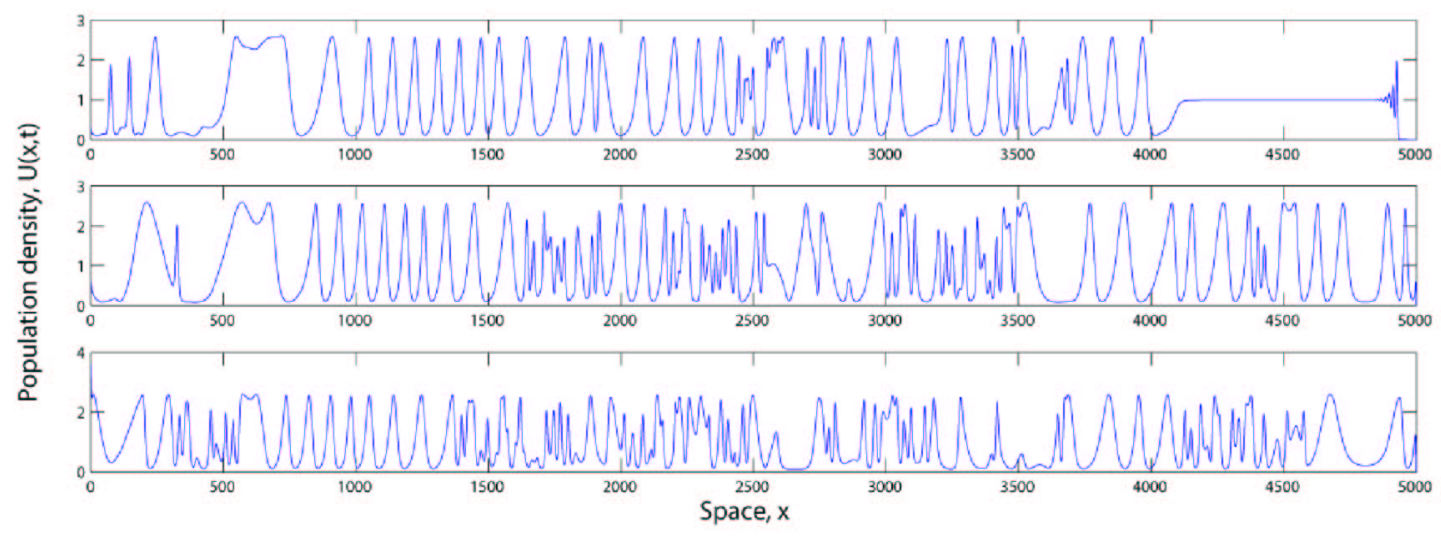

Figure 4.13: Snapshots of spatial propagation of population front with spatial averaging for longer simulation time. The time delay parameter is chosen to be 1.95 .

for the $1 \mathrm{D}$ case. The chosen kernel is thus normally distributed with a dependence on time delay. Our model now allows for the spatial drift of individuals and operates under the assumption that competition (which, in our model, is the biological mechanism of the time delay) does not occur instantaneously. Arguably, this increases the reality of the model and as such it is considered ecologically more viable.

It does not seem possible to establish the properties of model (4.23) analytically so we have to use numerical simulations. As before, the model (4.23) is approximated using finite differences with sufficiently small mesh steps. Neumann 'no flux' boundary conditions are implemented and the additional spatial average term (integral) is approximated by the trapezium rule. Numerical results are presented in Figs. 4.12-4.16. Interestingly, we observe that the qualitative dynamics have not been altered by changing the simple delayed logistic growth (as in Eq. (3.20)) to the 


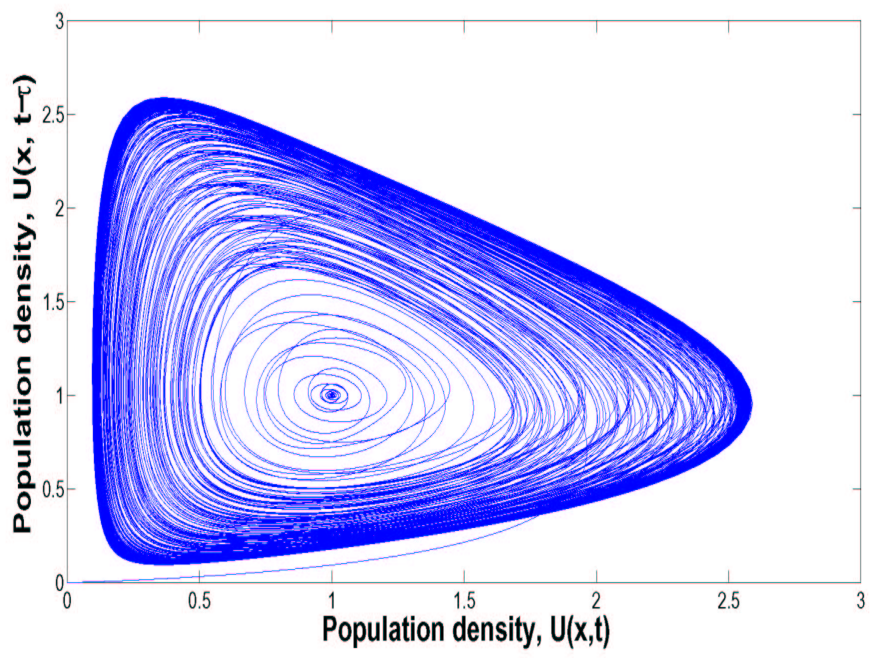

Figure 4.14: The phase plane $\left(U\left(x_{*}, t\right), U\left(x_{*}, t-\tau\right)\right)$ obtained at a fixed point in the middle of the domain for $\tau=2.0$.

more complicated spatial averaging term as in Eq. (4.23). The succession of regimes in response to an increase in $\tau$ remains the same. The initial conditions evolve to the propagating population front. For small values of the time delay $\left(0<\tau<\tau_{c r}\right)$ the front is monotone; for somewhat larger values $\left(\tau_{c r}<\tau<\tau_{H}\right)$ there are oscillations at the front that promptly decays to the uniform distribution $U(x, t)=1$ (not shown here for the sake of brevity). For the overcritical values of time delay, i.e. for $\tau>\tau_{H}$, the spatiotemporal chaos emerges at the wake of the front preceded by the quasi-uniform distribution (Figs. 4.12 and 4.13). For a longer time, the irregular spatiotemporal oscillations occupy the whole domain; see the middle and bottom panels in Fig. 4.13. The oscillations are self-organised and self-sustained. The irregularity of oscillations is also clearly seen in the corresponding phase plane (population densities recorded at a fixed spatial location $x_{*}$, separated by a time lag equal to the delay), cf. Fig. 4.14, hence suggesting that the dynamics is chaotic.

In order to provide a stronger evidence that the irregular oscillations are chaotic, we have tested the sensitivity of the solution to a small perturbation of the initial conditions. The results are shown in Fig. 4.15. It is readily seen that slightly perturbed initial conditions eventually result in a different solution, which is known to be a fingerprint of chaos. We therefore conclude that the irregular oscillations in the wake are indeed chaotic. Note that the observed chaos is essentially spatiotemporal as the corresponding non-spatial system cannot exhibit chaos but only periodical oscillations.

As well as in the diffusive Hutchinson model (3.20), for a somewhat higher value of $\tau$ the dynamics change, so that, instead of the unstable plateau, a regular wavetrain emerges behind the propagating front, which is followed by the spatiotemporal chaos in the wake; see Fig. 4.16. As well as above, this spatiotemporal pattern is self-organised and self-sustained, and arises as a consequence of the interplay between delay, spatial averaging and local interactions (intraspecific competition).

Thus, we observe that, qualitatively, the dynamics of the arguably more realistic model (4.23) 


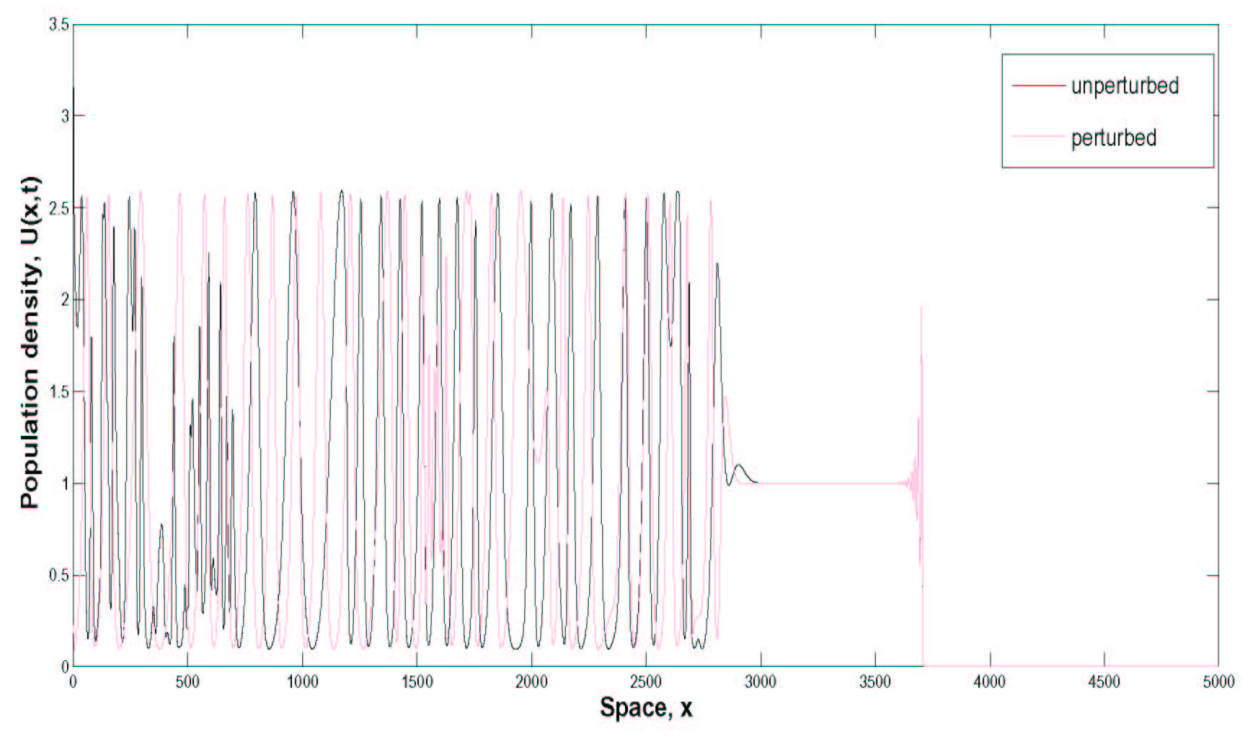

Figure 4.15: Sensitivity to the initial conditions for $\tau=1.7$ : the population fronts obtained from Eq. (4.23) with slightly different initial conditions, see Eqs. (3.21-3.22). The 'unperturbed' initial condition is taken as $U_{0}=0.5$ and the 'perturbed' one is $U_{0}=0.505$.

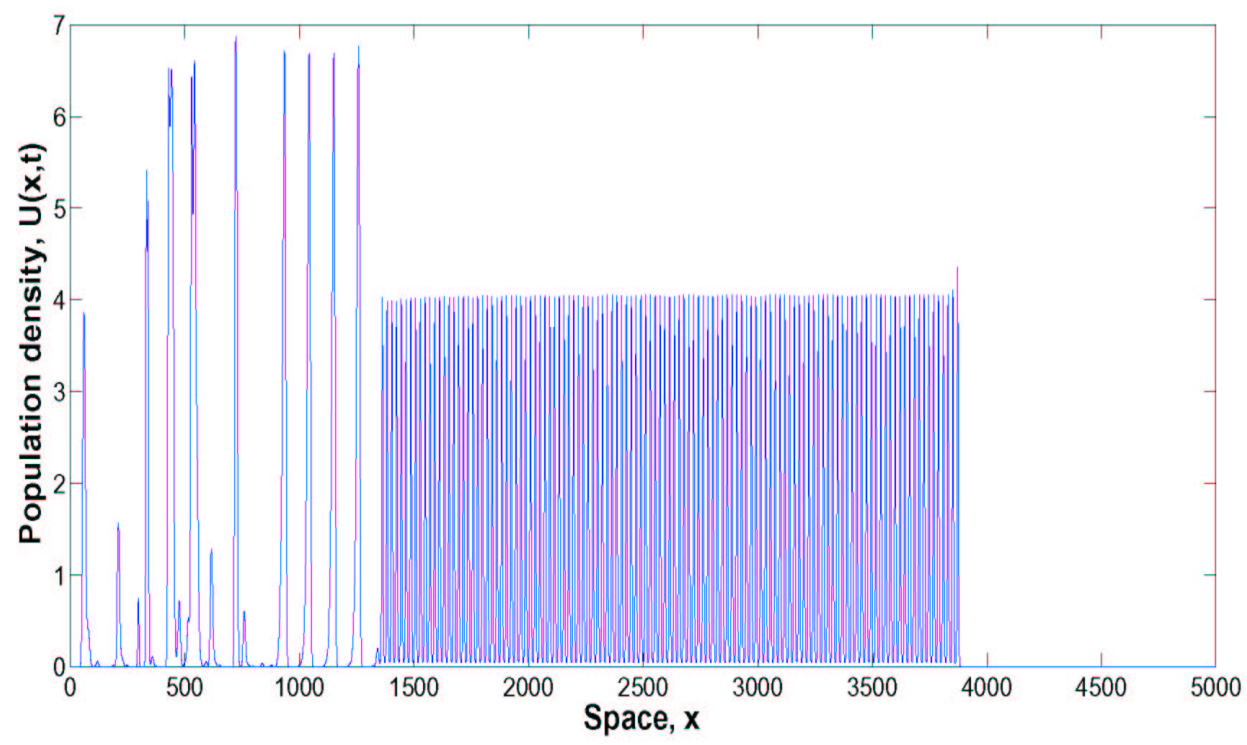

Figure 4.16: Spatial propagation of population front for $\tau=3$. The wavetrain preceding spatiotemporal chaos in the wake emerges.

is similar to that of the baseline model (2.4). The succession of the dynamical regimes that occurs as a response to the increase in time delay is essentially the same, i.e. from (i) a monotonous front to (ii) the stable plateau behind the oscillating front to (iii) the spatiotemporal chaos in the wake of the unstable plateau to, finally, (iv) regular wavetrain behind the front with chaos 
in the wake.

\section{Discussion and concluding remarks}

Factors and mechanisms resulting in complex spatiotemporal population dynamics and ecological pattern formation have been a major focus in ecology for several decades (Hassell et al. 1976, Turchin and Taylor 1992, Turchin 2003, Grünbaum 2012, van de Koppel 2008). There is a general understanding that spatial patterning usually results from a combination of exogenous and endogenous factors (Ranta et al. 1995, Rietkerk and van de Koppel 2007). The most obvious exogenous factor is the environmental heterogeneity which shapes the population dynamics through physical-chemical limitations (Kareiva et al. 1990). There is, however, considerable empirical evidence that heterogeneity alone is often not enough to explain observed heterogeneous population distribution (e.g. Powell et al. 1975, Sharov et al. 1997, Li et al. 2016). This eventually led to the concept of "self-organised" pattern formation, i.e. pattern formation due to (solely) endogenous factors that can, ultimately, take place in a perfectly uniform environment (Segel and Jackson 1972, Levin and Segel 1976, Okubo 1980, Okubo and Levin 2001, Petrovskii and Malchow 2001).

There is a considerable number of empirical studies on self-organised pattern formation in real ecological communities. Local interactions involving competition and facilitation have been shown to be the driving factor of regular spatial patterning in tussock Carex stricta in a freshwater wetland (van de Koppel and Crain 2006) and in larch budmoth in Alps (Bjørnstad et al. 2002). Self-organised vegetation patterns found in bogs, such as string and maze patterns, are thought to originate from a nutrient accumulation mechanism (Rietkerk et al. 2004). Experimental studies on coral reef fishes reveal that in some instances habitat is a prevalent determinant of spatial patterns, such as with damselfish, Dascyllus aruanus (Holbrook et al. 2000), and endogenous mechanisms such as recruitment patterns are responsible in others, as in populations of wrasse, Labridae (Green 1996). Self-organised patterns are noted in beds of blue mussel populations, Mytilus edulis, on intertidal flats with significant variability in mussel density observed on larger scales of meters, but with no apparent within-cluster difference. It is suggested that the interaction between individual mussels is the only underlying mechanism of such pattern formation (Gascoigne et al. 2005, van de Koppel et al. 2008). In the quest of revealing irregular spatial distributions, the most intuitive starting point would be amongst cyclic populations, as they tend to be regulated by highly nonlinear interactions (Bjørnstad et al. 2002).

Despite the abundant theoretical literature on self-organised pattern formation, there remain issues that are poorly understood. One such issue is the minimum level of system's complexity allowing for pattern formation with realistic properties. Pattern formation due to the Turing instability that normally results in a regular population spatial distribution (which is uncommon for animal species) was shown to require a minimum of two interacting species, with additional constraints requiring the species diffusivity to be different and the trophic interactions to be of activator-inhibitor type (Segel and Jackson 1972). Pattern formation due to a non-Turing mechanism dubbed "biological turbulence" or "wave of chaos" (Petrovskii and Malchow 2001), which results in somewhat more realistic irregular spatial distributions, was shown to be possible for equal diffusion coefficients but still require at least two interacting species (Sherratt et al. 1995, 
Petrovskii and Malchow 1999, Malchow et al. 2008). Here we have shown that, in fact, the presence of two interacting species is not necessary and pattern formation and rich population dynamics including spatiotemporal chaos is possible in a single species system continuous in space and time in case its reproduction is affected by a time delay.

Thus, the aim of this paper is to investigate the effect of space on the interplay between time delay and population growth in a single species system. We considered two different mathematical models. Firstly, we considered the delayed diffusive logistic population, i.e., essentially, the Hutchinson model with diffusion. We showed that travelling population fronts (which are generic for our choice of the initial population distribution) are capable of generating rich dynamics, resulting in an array of different spatiotemporal patterns for different values of the time delay. Note that the destabilising effect of time delays through the Hopf bifurcation is not new; however, the resulting spatiotemporal patterns are nontrivial. In particular, to the best of our knowledge, the onset of spatiotemporal chaos in the system (2.4) has not been reported before ${ }^{4}$. As the time delay, $\tau$, increases from zero, the population front loses monotonicity through the appearance of a single hump which evolves into damped oscillations. These oscillations increase in amplitude with increasing $\tau$, though decay promptly as the upper equilibrium $U_{*}=K$ is still stable (Fig. 3.4). For a sufficiently large $\tau$, irregular oscillations in the wake of the front appear (Figs. 3.5-3.6). These spatiotemporal patterns are self-organised, thus being an inherent property of the time delayed system. Connecting the damped oscillations at the leading edge and spatiotemporal patterns in the wake, there is an emerging quasi-homogeneous species distribution. Peculiarly, this plateau coincides with the otherwise unstable equilibrium. For a slight change in $\tau$, the plateau disappears to give way to periodic oscillations, cf. Figs. 3.6 and 3.7, that are followed by chaotic spatiotemporal oscillations. Note that the spatiotemporal chaos is self-sustained: following its onset and the subsequent eventual spread over the entire domain, no further qualitative changes appear in the system dynamics. The corresponding $2 \mathrm{D}$ system is shown to have similar dynamics (Fig. 3.11).

Secondly, we have considered a somewhat more realistic model that takes accounts for the fact that the interactions delayed in time may, due to the movement of individuals, have taken place at a different position in space (Britton 1990). Mathematically, the model is given by the integral-differential equation (4.23). Having studied the properties of Eq. (4.23) in extensive numerical simulations, we obtained that, with an increase in $\tau$, it shows a succession of dynamical regimes very similar to that of the standard model (2.4) (or (3.20) in dimensionless variables). Therefore, the inclusion of spatial averaging as a more realistic biological concept does not alter the observed succession of spatiotemporal dynamics. In particular, for sufficiently large values of $\tau$ we observe the dynamical stabilization of unstable equilibrium (plateau) behind the front followed by the onset of spatiotemporal chaos (Fig. 4.12). Spatiotemporal chaos is self-sustained and eventually occupies the whole domain (Fig. 4.13).

Note that, although the concept of spatiotemporal patterns in the wake of travelling fronts (in particular, the dynamical stabilization of an unstable equilibrium and spatiotemporal chaos) is by itself not new, it has usually been observed in multi-species models. Well-known examples are given by a predator-prey system (Sherratt 1994, Sherratt et al. 1995, Petrovskii and Mal-

\footnotetext{
${ }^{4}$ Ashwin et al. (2002) considered a similar system and concluded that "there is a region in which the solution develops irregular spatial fluctuations whilst apparently being periodic in time".
} 
chow 2000), a three-species competition system (Petrovskii et al. 2001) and a chemical activatorinhibitor system (Malchow and Petrovskii 2002). Here we have demonstrated that such patterns can arise in a single species population model due to the delayed density-dependent reproduction.

Our results seem to suggest a certain universality. The dynamics with essentially the same properties is observed in instantaneous reaction-diffusion systems (e.g. see the examples above) as well as in the delayed single species system in the parameter ranges of the same meaning, i.e. where the coexistence state becomes unstable through the supercritical Hopf bifurcation leading to the emergence of periodical temporal oscillations. However, the biological processes resulting in this self-organized periodicity are obviously very different in the single species population with a time delay in maturation and in a multispecies community without such delay. This observation suggests that the stable limit cycle is the defining feature of the system dynamics not only in time but also in space (at least in a certain parameter range not too far away from the Hopf bifurcation) regardless of the specific biological mechanisms that result in the emergence of the limit cycle.

\section{References}

[1] Adamson M, Morozov A Y (2012) Revising the role of species mobility in maintaining biodiversity in communities with cyclic competition. Bull Math Biol 74(9): 2004-2031.

[2] Ashwin P, Bartuccelli V, Bridges T J, Gourley S (2002) Travelling fronts for the KPP equation with spatio-temporal delay. Z angew Math Phys 53:102-122.

[3] Berryman A, Turchin P (1997) Detection of delayed density dependence: Comment. Ecology $78: 318-320$.

[4] Bjørnstad O N, Peltonen M, Liebhold A M, Baltensweiler W (2002) Waves of larch budmoth outbreaks in the European Alps. Science 298:1020-1023.

[5] Briggs C J, Sait S M, Begon M, Thompson D J, Godfray H C J (2000) What causes generation cycles in populations of stored-product moths? J Anim Ecol 69(2):352-366.

[6] Britton N F (1990) Spatial structures and periodic travelling waves in an integro-differential reaction-diffusion population model. SIAM J Appl Math 50(6):1663-1688.

[7] Busenberg S, Huang W (1996) Stability and Hopf bifurcation for a population delay model with diffusion effects. J Diff Eqs 124:80-107.

[8] Comins H N, Hassell M P (1976) Predation in multi-prey communities. J Theor Biol 62:93114.

[9] Davis M B, Calcote R R, Sugita S, Takahara H (1998) Patchy invasion and the origin of a Hemlock-Hardwoods forest mosaic. Ecology 79:2641-2659. 
[10] Elton C, Nicholson M (1942) The ten-year cycle in numbers of the lynx in Canada. J. Anim. Ecol. 11:215-244.

[11] Fortin MJ, Dale MRT (2005). Spatial Analysis: A Guide for Ecologists. Cambridge: Cambridge University Press.

[12] Fowler A C (1982) An asymptotic analysis of the delayed logistic equation when the delay is large. IMA J Appl Math 28:41-49.

[13] Gascoigne J C, Beadman H A, Saurel C, Kaiser M J (2005) Density dependence, spatial scale and patterning in sessile biota. O ecologia 145(3):371-381.

[14] Ginzburg L R, Taneyhill D E (1994) Population cycles of forest Lepidoptera: a maternal effect hypothesis. J Anim Ecol 63:79-92.

[15] Gourley S A , Bartuccelli M V (1995) Length scales in solutions of a scalar reaction-diffusion equation with delay. Phys Lett A 202:79-87.

[16] Gourley S A, Chaplain M A J (2002) Travelling fronts in a food-limited population model with time delay. Proc R Soc Edinburgh 132A:75-89.

[17] Green D, Stech H W (1981) Diffusion and hereditary effects in a class of population models. In Differential equations and applications in ecology, epidemics and population problems (Ed Busenberg S and Cooke K L) pp. 19-28. Academic Press, New York.

[18] Grünbaum D (2012) The logic of ecological patchiness. Interface Focus 2, 150-155.

[19] Hansen T F, Stenseth N C, Henttonen H, Tast J (1998) Interspecific and intraspecific competition as causes of direct and delayed density dependence in a fluctuating vole population. Proc Natl Acad Sci USA 96(3):986-991.

[20] Hassell M P, Lawton J H, May R M (1976) Patterns of dynamical behaviour in single species populations. Journal of Animal Ecology 45:471-486.

[21] Hassell M P, Comins H N, May R M (1991) Spatial structure and chaos in insect population dynamics. Nature 353:255-258.

[22] Hastings A (1984) Delays in recruitment at different trophic levels: Effects on stability. Journal of Mathematical Biology 21:35-44.

[23] Hastings A, Harisson S, McCann K (1997) Unexpected spatial patterns in an insect outbreak match a predator diffusion model. Proc. R. Soc. Lond. B 264:1837-1840.

[24] Holt RD (1983) Optimal foraging and the form of the predator isocline. Amer Nat 122:521541.

[25] Huang W (1998) Global dynamics for a reaction-diffusion equation with time delay. Journal of Differential Equations 143:293-326.

[26] Hutchinson G E (1948) Circular causal systems in ecology. Annals of the New York Academy of Science 50:221-246. 
[27] Jankovic M, Petrovskii S (2013) Gypsy moth invasion in North America: A simulation study of the spatial pattern and the rate of spread. Ecological Complexity 14:132-144.

[28] Jankovic M, Petrovskii S (2014) Are time delays always destabilizing? Revisiting the role of time delays and the Allee effect. Theor Ecol 7:335-349.

[29] Kareiva P, Mullen A, Southwood R (1990) Population dynamics in spatially complex environments: theory and data (and discussion). Phil. Trans. R. Soc. Lond. B 330(1257):175-190.

[30] Klausmeier C A (1999) Regular and irregular patterns in semiarid vegetation. Science $284: 1826-1828$.

[31] Levin S (1994) Patchiness in marine and terrestrial systems: from individuals to populations. Phil. Trans. R. Soc. Lond. B 343:99-103.

[32] Levin S A, Segel L A (1976) Hypothesis for origin of planktonic patchiness. Nature 259:659.

[33] Li K, Vandermeer J H, Perfecto I (2016) Disentangling endogenous versus exogeenous pattern formation in spatial ecology: A case study of the ant Azteca sericeasur in southern Mexico. R. Soc. Open Sci., submitted.

[34] Li W T, Yan X P, Zhang C H (2008) Stability and Hopf bifurcation for a delayed cooperation diffusion system with Dirichlet boundary conditions. Chaos, Solitons and Fractals 38:227237.

[35] Liebhold A M, Elmes G A, Hawerson J A, Quimby J (1994) Landscape characterization of forest susceptibility to gypsy moth defoliation. Forest Science 40:18-29.

[36] Liebhold AM, McCullough DG, Blackburn LM, Frankel SJ, von Holle B, Aukema JE (2013) A highly aggregated geographical distribution of forest pest invasions in the USA. Diversity Distrib 2013;19(9):1208-16.

[37] Malchow H, Petrovskii S V (2002) Dynamical stabilisation of an unstable equilibrium in chemical and biological systems. Mathematical and Computer Modelling 36:307-319.

[38] Malchow H, Petrovskii S V, Venturino E (2008) Spatiotemporal patterns in ecology and epidemiology: Theory, Models and Siulation. Chapman \& Hall/CRC.

[39] Martin A P (2003) Phytoplankton patchiness: the role of lateral stirring and mixing. Progress in Oceanography 57:125-174.

[40] May R M (1972) Limit cycles in predator-prey communities. Science 177:900-902.

[41] May R M (1975) Stability and complexity in Model Ecosystems. Princeton University Press. Princeton, second edition.

[42] Medvinsky A B, Petrovskii S V, Tikhonova I A, Malchow H, Li B.-L (2002) Spatiotemporal complexity of plankton and fish dynamics. SIAM Review 44:311-370.

[43] Mimura M, Tohma M (2014) Dynamic coexistence in a three-species competitionæİAiffusion system. Ecol. Complex., in press, http://dx.doi.org/10.1016/j.ecocom.2014.05.004 
[44] Mogilner, A., Edelstein-Keshet L (1999) A non-local model for a swarm. Journal of Mathematical Biology 38:534Ü570.

[45] Mogilner A, Edelstein-Keshet L, Bent L, Spiros A (2003) Mutual interactions, potentials, and individual distance in a social aggregation. J. Math. Biol. 47(4):353-389.

[46] Morozov A, Petrovskii S V (2013) Feeding on multiple sources: towards a universal parameterization of the functional response of a generalist predator allowing for switching. PLoS One 8(9):e74586.

[47] Murray J D (2002) Mathematical Biology I: an introduction. Springer.

[48] Okubo A (1986) Dynamical aspects of animal grouping: swarms, schools, flocks and herds. Adv. Biophys. 22:1-94.

[49] Okubo A (1980) Diffusion and ecological problems: mathematical models. Springer-Verlag, Berlin

[50] Okubo A, Levin S (2001) Diffusion and Ecological Problems: Modern Perspectives. Springer, Berlin.

[51] Petrovskii S V, Malchow H (1999) A minimal model of pattern formation in a prey-predator system. Mathematical and Computer Modelling 29(8):49-63.

[52] Petrovskii S V, Malchow H (2000) Critical phenomena in plankton communities: KISS model revisited. Nonlinear Analysis: Real World Applications 1:37-51.

[53] Petrovskii S V, Malchow H (2001) Wave of chaos: new mechanism of pattern formation in spatio-temporal population dynamics. Theoretical Population Biology 59:157-174.

[54] Petrovskii S V, Li B.-L, Malchow H (2003) Quantification of the spatial aspect of chaotic dynamics in biological and chemical systems. Bulletin of Mathematical Biology 65:425-446.

[55] Petrovskii S, Kawasaki K, Takasu F, Shigesada N (2001) Diffusive waves, dynamical stabilization and spatio-temporal chaos in a community of three competitive species. Jpn. J. Ind. Appl. Math. 18:459-481.

[56] Powell T M, Richerson P J, Dillon T M, Agee B A, Dozier B J, Godden D A, Myrup L $\mathrm{O}(1975)$ Spatial scales of current speed and phytoplankton biomass fluctuations in Lake Tahoe. Science 189:1088-1090.

[57] Ranta E, Vaitala K, Lindstrom J, Linden H (1995) Synchrony in population dynamics. Proc. R. Soc. Lond. B 262(1364):113-115.

[58] Rietkerk M, Dekker S C, de Ruiter P C, van de Koppel J (2004) Self-organized patchiness and catastrophic shifts in ecosystems. Science 305: 1926-1929.

[59] Rietkerk M, van de Koppel J (2007) Regular pattern formation in real ecosystems. Trends in Ecology and Evolution 23(3):169-175. 
[60] Ritchie M E (2010) Scale, heterogeneity and the structure and diversity of ecological communities. Princeton, NJ: Princeton University Press.

[61] Rosenzweig M L (1971) Paradox of enrichment: Destabilization of exploitation ecosystems in ecological time. Science 171:385-387.

[62] Royama T (1981) Fundamental concepts and methodology for the analysis of animal population dynamics, with particular reference to univoltine species. Ecological Monographs 51:473-493.

[63] Segel L A, Jackson J L (1972) Dissipative structure: an explanation and an ecological example. J. Theor. Biol. 37:545-559.

[64] Sharov A A, Liebhold A M, Roberts E A (1997) Correlation of counts of gypsy moths (Lepidoptera: Lymantriidae) in pheromone traps with landscape characteristics. Forest Science 43 (3): 483-490.

[65] Sherratt J A (1994) Irregular wakes in reaction-diffusion waves. Physica D 70:370-382.

[66] Sherratt J A (2001) Periodic travelling waves in cyclic predator-prey systems. Ecol. Lett. $4: 30-37$.

[67] Sherratt J A (2013) Pattern solutions of the Klausmeier model for banded vegetation in semi-arid environments V: the transition from patterns to desert. SIAM J. Appl. Math. 73:1347-1367.

[68] Sherratt J A, Smith M J (2008) Periodic travelling waves in cyclic populations: field studies and reaction-diffusion models. J R Soc Interface 5:483-505.

[69] Sherratt J A, Lewis M A, Fowler A (1995) Ecological chaos in the wake of invasion. Proc Natl Acad Sci USA 92:2524-2528.

[70] Shigesada N, Kawasaki K (1997) Biological Invasions: Theory and Practice. Oxford University Press, Oxford.

[71] Su Y, Wei J, Shi J (2009) Hopf bifurcations in a reaction diffusion population model with delay effect. Journal of Differential Equations 247:1156-1184.

[72] Turchin P (2003) Complex population dynamics: A theoretical/empirical synthesis. Princeton University Press.

[73] Turchin P, Taylor A D (1992) Complex dynamics in ecological time series. Ecology 73(1):289305.

[74] Tyutyunov YV, Senina I, Arditi R (2004) Clustering due to acceleration in the response to population gradient: a simple self-organization model. Am. Nat. 164(6):722-735.

[75] Tyutyunov YV, Zagrebneva AD, Surkov FA, Azovsky AI (2009) Microscale patchiness of the distribution of copepods (Harpacticoida) as a result of trophotaxis. Biophysics 54(3):508514. 
[76] Van de Koppel J, Gascoigne J C, Theraulaz G, Rietkerk M, Mooij W M, Herman P M J (2008) Experimental evidence for spatial self-organisation and its emergent effects in mussel bed ecosystems. Science 322(5902):739-742.

[77] Van Leeuwen E, Brännström Å, Jansen V A, Dieckmann U, Rossberg A G (2013) A generalized functional response for predators that switch between multiple prey species. J Theor Biol 328: 89-98.

[78] Yoshida K (1982) The Hopf bifurcation and its stability for semilinear diffusion equations with time delay arising in ecology. Hiroshima Mathematical Journal 12:321-348. 